\title{
Comparable exposure to SARS-CoV-2 in young children and
}

\section{healthcare workers in Zambia [version 1; peer review: 2}

\section{approved with reservations]}

\author{
Natasha Makabilo Laban(i1,2, Samuel Bosomprah1,3, Kalo Musukuma-Chifulo(D1, \\ Michelo Simuyandi (D1, Shilpa Iyer 1,4, Harriet Ng'ombe1, Mutinta Muchimba1, \\ Adriace Chauwa1, Sekayi Tigere ${ }^{1}$, Caroline Cleopatra Chisenga(D1, \\ Mwelwa Chibuye1, Obvious Nchimunya Chilyabanyama (D1), Martin Goodier(D), \\ Roma Chilengi (D1,4
}

\footnotetext{
${ }^{1}$ Research Department, Centre for Infectious Disease Research in Zambia, Lusaka, 10101, Zambia

${ }^{2}$ Department of Infection Biology, London School of Hygiene \& Tropical Medicine, London, WC1E 7HT, UK

${ }^{3}$ Department of Biostatistics, University of Ghana, Accra, Ghana

${ }^{4}$ School of Medicine, University of Alabama at Birmingham, Birmingham, USA
}

\author{
V1 First published: 06 May 2021, 6:97 \\ https://doi.org/10.12688/wellcomeopenres.16759.1 \\ Latest published: 06 May 2021, 6:97 \\ https://doi.org/10.12688/wellcomeopenres.16759.1
}

\section{Abstract}

Background: Coronavirus disease 2019 (COVID-19) caused by severe acute respiratory syndrome coronavirus 2 (SARS-CoV-2) is an ongoing global health crisis that has caused large scale morbidity and mortality. We aimed to determine the exposure to SARS-CoV-2 among young children and healthcare workers by measurement of anti-S1 antigen (spike protein) specific immunoglobulin G (IgG) using an inhouse optimized indirect enzyme-linked immunosorbent assay (ELISA) method.

Methods: Plasma samples were collected from cohorts of healthcare workers $(n=287)$ and young children aged from 6 weeks to 2 years old $(n=150)$ pre-COVID-19 pandemic between September 2018 and November 2019 and post-COVID-19 pandemic between August and December 2020 were simultaneously tested for anti-SARS-CoV-2 S1 specific IgG. The arithmetic mean of natural logarithm-transformed ELISA relative absorbance reading + ( $3 \times$ standard deviation) of prepandemic plasma was used as the cut-off to determine SARS-CoV-2 IgG seropositivity of post-pandemic plasma.

Results: There was no reactivity to SARS-CoV-2 S1 antigen detected in pre-pandemic plasma but in post pandemic plasma an $8.0 \%(23 / 287)$ IgG seropositivity in healthcare workers' and 6.0\% (9/150) seropositivity in children aged 2 years old was detected.

Conclusions: Comparable levels of SARS-CoV-2 IgG seropositivity in healthcare workers and children suggest widespread exposure to

\section{Open Peer Review}

\begin{tabular}{lcc} 
Approval Status & ? ? \\
& 1 & 2 \\
\hline version 1 & $?$ & $?$ \\
06 May 2021 & view & view
\end{tabular}

1. Elizabeth Mayne, University of Witwatersrand, Johannesburg, South Africa

2. Khitam Muhsen ID, Tel Aviv University, Tel Aviv, Israel

Any reports and responses or comments on the article can be found at the end of the article. 
SARS-CoV-2 in Zambia during the first wave of the pandemic. This finding has implications for continued acquisition and transmission of infection in the healthcare setting, household, and wider community.

\section{Keywords}

Anti-S1 IgG, children, ELISA, healthcare workers, SARS-CoV-2, Zambia, seroprevalence

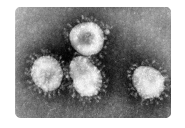

This article is included in the Coronavirus

(COVID-19) collection.

Corresponding author: Natasha Makabilo Laban (Natasha.Laban@cidrz.org)

Author roles: Laban NM: Funding Acquisition, Investigation, Project Administration, Validation, Writing - Original Draft Preparation, Writing - Review \& Editing; Bosomprah S: Data Curation, Formal Analysis, Methodology, Software, Visualization, Writing - Review \& Editing; Musukuma-Chifulo K: Investigation, Writing - Review \& Editing; Simuyandi M: Supervision, Writing - Review \& Editing; Iyer S: Writing - Review \& Editing; Ng'ombe H: Investigation; Muchimba M: Investigation; Chauwa A: Investigation; Tigere S: Investigation; Chisenga CC: Supervision, Writing - Review \& Editing; Chibuye M: Data Curation, Formal Analysis, Visualization, Writing - Review \& Editing; Chilyabanyama ON: Data Curation, Formal Analysis, Visualization, Writing - Review \& Editing; Goodier M: Conceptualization, Methodology, Resources, Writing - Review \& Editing; Chilengi R: Conceptualization, Funding Acquisition, Resources, Writing - Review \& Editing

Competing interests: No competing interests were disclosed.

Grant information: This work was supported through funds from a Wellcome Trust International Training Fellowship [211356/Z/18/Z] awarded to NL. The funders had no role in the actual design, execution and interpretation of data, and the views and conclusions expressed in this article are the authors' only.

The funders had no role in study design, data collection and analysis, decision to publish, or preparation of the manuscript.

Copyright: ( 2021 Laban NM et al. This is an open access article distributed under the terms of the Creative Commons Attribution License, which permits unrestricted use, distribution, and reproduction in any medium, provided the original work is properly cited.

How to cite this article: Laban NM, Bosomprah S, Musukuma-Chifulo $\mathrm{K}$ et al. Comparable exposure to SARS-CoV-2 in young children and healthcare workers in Zambia [version 1; peer review: 2 approved with reservations] Wellcome Open Research 2021, 6:97 https://doi.org/10.12688/wellcomeopenres.16759.1

First published: 06 May 2021, 6:97 https://doi.org/10.12688/wellcomeopenres.16759.1 


\section{Introduction}

Coronavirus disease 2019 (COVID-19) caused by the novel severe acute respiratory syndrome coronavirus 2 (SARS-CoV-2) is an ongoing global public health emergency that as of April 2021 has infected more than 100 million people and claimed nearly three million lives worldwide ${ }^{1}$. While reported incidence and mortality has been strikingly lower in Africa compared to other regions of the world, Africa within this period had still recorded over three million cases with over 70,000 deaths ${ }^{1}$. In Zambia, the first confirmed case of SARS-CoV-2 was reported on $18^{\text {th }}$ March, 2020 and since then, over 90,000 confirmed cases and more than 1,200 deaths have been reported as of April 20212,3. The robust global response to the pandemic has seen unprecedented advancements in research leading to elucidation of the SARS-CoV-2 genome ${ }^{4}$, development of diagnostic tests and the accelerated development of vaccines in $<12$ months since the SARS-CoV-2 outbreak was first reported ${ }^{5,6}$ with more than 600 million vaccine doses administered by April $2021^{1}$.

Nucleic acid-based testing is the current diagnostic test of choice for active SARS-CoV-2 infection but high costs and the need for specific sample collection and transport conditions, specialised laboratory facilities and skilled personnel limit accessibility and feasibility for large scale screening. In the absence of mass SARS-CoV-2 nucleic acid testing for disease surveillance particularly in lower income settings, the true extent of SARS-CoV-2 exposure within high-risk populations such as frontline healthcare workers and the wider population may be underestimated. In such settings, serology can serve as an important complementary diagnostic and surveillance tool ${ }^{7,8}$. Locally developed serological tests like the antibody-based immunoassays capable of effectively discriminating SARSCoV-2 exposure by detection of specific immunoglobulins ${ }^{9,10}$ are important for both immunosurveillance ${ }^{8}$ and evaluating vaccine responses post implementation.

An intriguing observation during the ongoing pandemic apart from the observed lower incidence and mortality rates in Africa was that the paediatric population recorded lower SARS-CoV-2 incidence, severity and mortality compared to older age groups, a phenomenon also seen in Zambia ${ }^{11,12}$. Although representing only a small fraction of confirmed COVID-19 cases, children are susceptible to SARS-CoV-2 infection with a substantial proportion of infected children tending to be asymptomatic and potentially missed in symptom-based case detection strategies ${ }^{13}$. Children have mild disease that delays detection and can exhibit prolonged SARS-CoV-2 viral shedding regardless of symptomatology ${ }^{14}$. Children could therefore act as a hidden infectious reservoir for household and community transmission networks and thus, it is important to understand the epidemiology of SARS-CoV-2 infection in this population.

Our objectives were to develop and optimize an in-house semi quantitative, indirect enzyme-linked immunosorbent assay (ELISA) for immunoglobulin $\mathrm{G}$ ( $\mathrm{IgG}$ ) directed against SARS-CoV-2 spike glycoprotein (S1) and screen stored plasma samples from a cohort of healthcare workers and young children to permit estimation of SARS-CoV-2 seroprevalence within the healthcare setting and among young children residing in a peri urban community in Zambia.

\section{Methods}

\section{Ethical considerations}

This study protocol including a request for waiver of participant informed consent to utilize samples collected from existing studies was approved by the National Health Research Authority based on the existing participant informed consent and ethical clearance by the University of Zambia Biomedical Research Ethics Committee (UNZABREC) for the Hepatitis B vaccine study (UNZABREC Reference Number 003-01-19) and rotavirus vaccine trial (UNZABREC Reference Number 003-02-18).

\section{Study setting and participants}

This study included healthcare workers that were enrolled in an existing "before and after" interventional study aimed at demonstrating healthcare worker's protection against Hepatitis $\mathrm{B}$ infection by vaccination ${ }^{15}$. The Hepatitis B vaccine study was conducted in Kalulushi district on the Copperbelt province of Zambia having a projected total population of approximately 140,000 in $2019^{16}$. Kalulushi district has nine health posts, eleven clinics and one second level hospital as well as one nursing school, one health training institute and university. The district had an estimated 405 healthcare workers with an additional 407 students and lecturers in health training institutions (Data sourced from Kalulushi District Health Office, 2019). The sample size for the Hepatitis B vaccine study was calculated based on confidence interval for one proportion and required a minimum sample size of 513 healthcare workers to estimate the prevalence of Hepatitis B with a 95\% confidence interval and 5\% width interval assuming a prevalence of $8.6 \%{ }^{17,18}$. The sample size was adjusted by $25 \%$ to control for losses to follow-up and secondary analyses. Before enrolment, sensitization talks about the Hepatitis B vaccine study were conducted by the study team at the health facilities and health professional schools after which eligibility was determined and written informed consent sought from those healthcare workers that were willing to participate. Inclusion criteria for the healthcare workers were individuals formally employed within a public health facility or students enrolled at an allied healthcare training institution in the district, aged $\geq 18$ years and willing to consent and meet study training and follow up requirements. Refusal to consent, pregnancy, yeast allergy, hypersensitivity, acute febrile illness and receipt of steroids, immune modulators or blood products were exclusionary criteria. A total of 641 healthcare workers were recruited using this approach and the enrolled healthcare workers were followed up from November 2019 through August 2020.

Paediatric plasma samples included in this study were from an ongoing open label randomised controlled rotavirus vaccine trial comparing two versus three dose vaccination in Lusaka, Zambia ${ }^{19}$. The outcome of the rotavirus vaccine study was boosted antibody responses after administration of the third vaccine dose in the intervention arm. The study determined 
the sample size based on an anticipated $15 \%$ or greater increase in rotavirus antibody response after the third dose and required a total of 196 infants (98 infants per arm) to detect this boosted response at an $80 \%$ power using two-sample t-test, and assuming equal standard deviation at 5\% level of significance. A $10 \%$ upward sample size adjustment was made to account for losses in follow up to reach a total of 214 children that were enrolled from a population residing in "George compound", a peri-urban unplanned settlement in Lusaka. Children aged between 6 to 12 weeks old attending the George public health facility with their mothers were targeted for enrolment. Mother-child pairs were approached during their visit to the maternal and child health department by the study Research Nurse and provided with sensitization information about the study in the mother's local language of choice. Mothers that were interested were invited to the study research unit co-located within the health facility premises for more detailed information during which willing mothers were checked for eligibility and taken through the written informed consent process. Criteria for enrolment included the mothers' willingness to participate voluntarily and provide consent, child's' eligibility for Rotarix vaccination as per national policy and mothers' willingness for child to undergo defined study procedures and residence in the study area for the duration of the study. Contraindication to rotavirus vaccination, previous receipt of rotavirus vaccine, recent immunosuppressive therapy including high-dose systemic corticosteroids, history of receiving blood transfusion or blood products, including immunoglobulins within the previous 6 months, any condition deemed by the study investigator to pose potential harm to the child or jeopardize the validity of study result and any existing congenital anomalies constituted exclusionary criteria. Eligible, consenting mothers were enrolled and followed up until the child attained 2 years of age.

\section{Data collection}

From the enrolled healthcare workers under the Hepatitis $B$ vaccine study, demographic data and pre-vaccination plasma samples were collected at baseline after written informed consent was obtained. Additional plasma samples were collected at subsequent scheduled appointments after vaccination during the follow-up period. In our study we utilized the healthcare worker pre-vaccination baseline plasma collected prior to declaration of the COVID-19 pandemic between November and December 2019 and the matched post-vaccination plasma samples that were collected in August 2020 during the COVID-19 pandemic.

Similarly, in the rotavirus vaccine trial mother-child cohort, demographic data and pre-vaccination plasma was collected at enrolment after mother's written informed consent was obtained. During the follow up period, additional plasma samples from the children were collected post-vaccination during scheduled study clinic appointments. For the present study, we used pre-vaccination baseline plasma collected when children were aged 6 to 12 weeks old between September and November 2018 (pre-COVID-19 pandemic) and matched plasma collected in the follow up period between August and December 2020 during the COVID-19 pandemic when children were aged 2 years old. Plasma samples from both cohorts were stored at $-20^{\circ} \mathrm{C}$ prior to serological testing in this study.

\section{Laboratory procedures}

Set up and optimisation of in-house ELISA. To develop our SARS-CoV-2 ELISA, we tested sero-reactivity of plasma against commercially available SARS-CoV-2 Spike/S1-His recombinant protein (Cat 40591-V08H, Sino Biologicals, China) using an indirect ELISA procedure. Seropositive plasma controls for anti-SARS-CoV-2 IgG were selected from known convalescent local Zambian adults previously tested and confirmed to be SARS-CoV-2 positive by polymerase chain reaction. Prospective adult donors were approached by the study team with information about the study and a request for plasma samples. Plasma was obtained from five individuals that were willing and provided consent to have a blood sample drawn. In addition to these seropositive controls, pre-pandemic plasma from five mothers among the mother-child cohort were used as seronegative plasma controls during initial experiments.

In these initial experiments we tested individual and pooled seropositive and seronegative control plasma including a blank (1\% skim milk in phosphate buffer, Blotto) control in the indirect ELISA assay method. To optimise the assay, we tested varying antigen coating concentrations, plasma sample dilutions, detection antibody concentrations, assay blocking solution and test sample incubation conditions. Briefly, microtiter plate wells were incubated with $50 \mu \mathrm{L}$ SARS-CoV-2 S1 antigen at three different concentrations $(0.5 \mu \mathrm{g} / \mathrm{ml}, 1 \mu \mathrm{g} / \mathrm{ml}$ and $2 \mu \mathrm{g} / \mathrm{ml})$ and blocked with different volumes of blotto $(150 \mu \mathrm{L}$ or $200 \mu \mathrm{L}$ ) for three hours at room temperature. Plasma sample controls at different dilutions (1:40 through 1:6400) and blotto (as blank to control for background absorbance) were then added in duplicate wells and incubated for either 2 hours at $37^{\circ} \mathrm{C}$ or overnight at $4{ }^{\circ} \mathrm{C}$. Peroxidase-conjugated anti-human IgG detection antibody at two dilutions (1:10000 or 1:15000) was added to the wells before incubation for three hours at room temperature, followed by addition of substrate, colour development and absorbance optical density (OD) reading at $492 \mathrm{~nm}$. Prior to each incubation step, the plate was placed on an incubator shaker set at $20^{\circ} \mathrm{C}, 270$ revolutions per minute for 20 minutes. Plate was also washed in between assay reagent and sample addition steps. We set our optimized ELISA as the assay with parameters yielding a $>1.5$ absorbance OD reading value for the pooled seropositive plasma and a 3-fold or more lower OD value for the seronegative control and blank. Based on these experiments, our optimized indirect ELISA required $1 \mu \mathrm{g} / \mathrm{ml}$ antigen coating concentration, $200 \mu \mathrm{L}$ blocking buffer volume, 1:100 sample dilution and overnight incubation and 1:15000 peroxidase-conjugated anti-human $\operatorname{IgG}$ antibody dilution. The optimisation experiments additionally determined a donor positive control sample with the highest SARS-CoV-2 $\mathrm{IgG}$ titer which was assigned as an in-house standard. These optimized conditions and procedures were used in all the subsequent assays.

Determination of SARS-CoV-2 IgG antibodies by ELISA. Healthcare workers' and children's plasma samples were tested 
using the optimized indirect ELISA. Briefly, a $50 \mu \mathrm{L}$ volume of $1 \mu \mathrm{g} / \mathrm{ml}$ SARS-CoV-2 antigen concentration in sodium carbonate-bicarbonate coating buffer at $\mathrm{pH} 9.6 \pm 0.2$ was added to the microtiter plate wells (Ref 655061, Greiner Bio-One) and incubated overnight at $4^{\circ} \mathrm{C}$. The following day, the plate was blocked with $200 \mu \mathrm{L} 1 \%$ blotto, weight/volume skim milk in phosphate-buffered saline containing $0.25 \%$ volume/volume Tween20 (Cat P1379, Sigma-Aldrich) (PBST), and incubated at room temperature for 3 hours. A $5 \mu \mathrm{L}$ volume of pre- and post-pandemic matched plasma sample diluted in a two-step wise manner in PBST and blotto to a final 1:100 was added in duplicate wells and incubated at $4^{\circ} \mathrm{C}$ overnight. On the third day, the plate was incubated with $50 \mu \mathrm{L}$ of peroxidase-conjugated goat anti-human IgG (Cat A0170, Sigma-Aldrich) diluted 1:15000 in PBST for 3 hours at room temperature. The colour reaction was developed using $50 \mu \mathrm{L}$ o-Phenylenediamine dihydrochloride substrate (Cat P4664, Sigma-Aldrich) diluted in citric acid phosphate buffer containing $0.03 \%$ volume/volume $30 \%$ hydrogen peroxide and stopped by addition of $100 \mu \mathrm{L} 1 \mathrm{M}$ Sulphuric acid before reading absorbance optical density (OD) at $492 \mathrm{~nm}$. Throughout the assay, wells were washed five times with PBST in between the ELISA steps. In each experiment, eight two-fold dilutions $(1: 100$ to $1: 12800)$ of the in-house SARS-CoV-2 IgG standard plasma, pooled seropositive and seronegative control plasma and blank (blotto) were included in duplicate wells. The pre-pandemic plasma samples were treated as internal negative controls to the matched post-pandemic samples for each participant in both the study cohorts.

\section{Statistical analysis}

Key background characteristics of healthcare workers and children were summarised using frequency and percentage for categorical variables, whereas median and interquartile range (IQR) were used to summarise age. Absorbance levels were calculated for each assay plate as relative absorbance units (rAU) with reference to values obtained for an in-house, high-titer standard plasma (assigned a value of $1000 \mathrm{rAU}$ ). To define seropositivity for SARS-CoV-2 IgG, we calculated a cut-off as the arithmetic mean of natural logarithm-transformed mean blank corrected arbitrary rAU of all pre-pandemic samples tested $+(3 \mathrm{x}$ standard deviation of the natural logarithm-transformed mean blank corrected arbitrary rAU). Seroprevalence was calculated as the proportion of samples with natural-log- arbitrary rAU above the calculated cut-off for the respective cohort. Fisher's exact test was used to assess the association of seroprevalence and key background characteristics. Dot plots and range plots were used to characterise the arbitrary rAU of samples in the two periods (pre- and post-SARS-CoV-2 pandemic). All analyses were performed using Stata 16 MP4 (StataCorp, College Station, TX, USA).

\section{Results}

A total of 287 healthcare workers were included in this study and had their pre- and post-pandemic matched plasma tested (Figure 1$)^{20}$. Included healthcare workers had a median age of 29 years (interquartile range 25, 37), were mostly female $187 / 287$ (65.2\%), had normal body mass index 149/287 (51.9\%) and were unmarried 145/287 (50.5\%). Among these healthcare workers most were medical staff 131/287 (45.6\%), while 100/287 (34.8\%) were support staff and 53/287 (18.5\%) were students.

A cut-off of 5.5381 arbitrary rAU was calculated from the healthcare worker pre-pandemic plasma and was used to
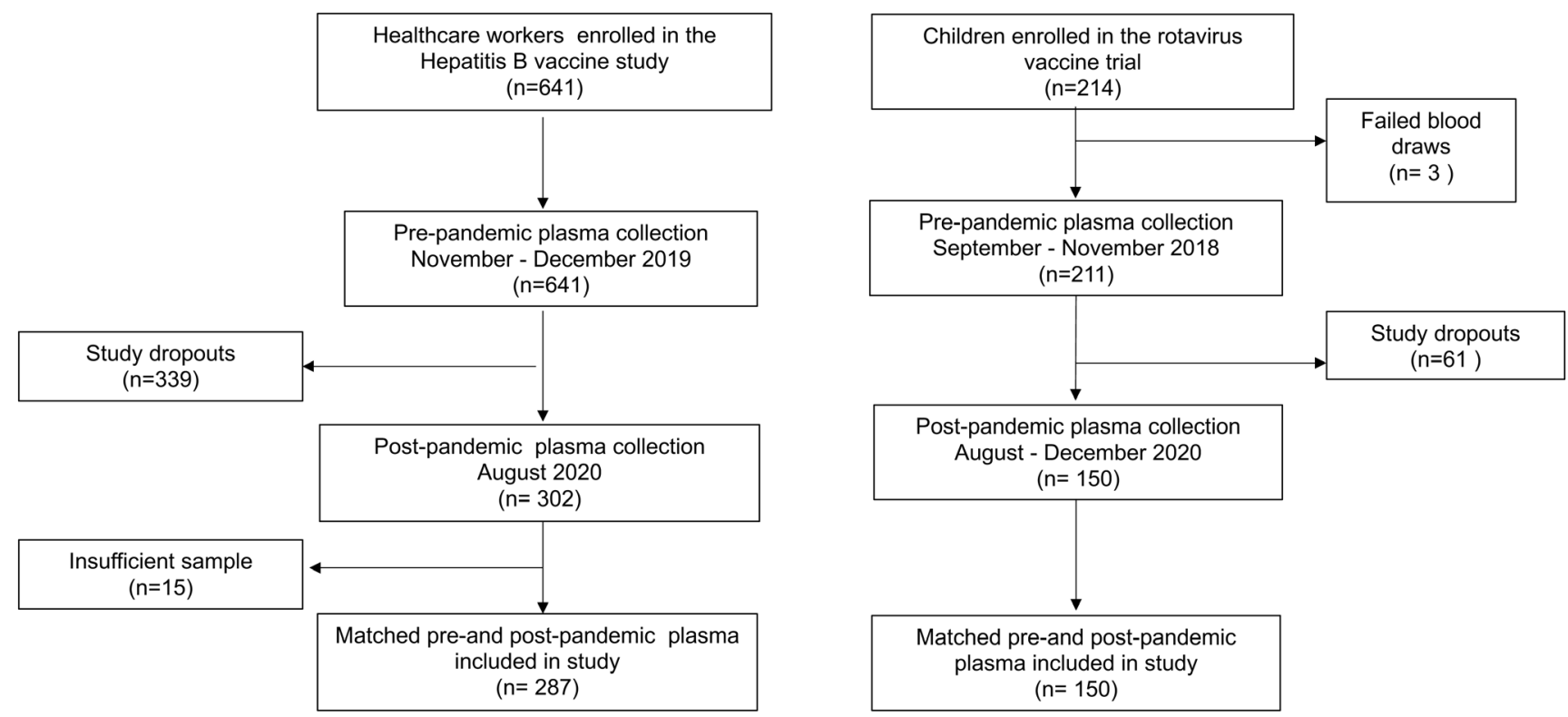

Figure 1. Selection of healthcare worker and child plasma samples included in the study. The study included stored matched plasma samples collected before the coronavirus disease 2019 (COVID-19) pandemic between September 2018 and December 2019 and post-pandemic between August and December 2020 from a cohort of healthcare workers $(n=287)$ recruited under a Hepatitis B vaccine study and children $(n=150)$ recruited under a rotavirus vaccine clinical 
determine seropositivity for this cohort. Overall, 23/287 healthcare workers were reactive for SARS-CoV-2 IgG above the calculated cut-off giving $8.0 \%$ seropositivity. Among these seropositives, most were among medical staff particularly among Nurses and Mid-wives. We did not find an association between any background characteristic included and seropositivity for SARS-CoV-2 IgG (Table 1).

We included and tested pre- and post-pandemic plasma from a total of 150 children in this study (Figure 1). These children had a median age of 6 weeks (interquartile range 6,6), were mostly male 84/150 (56\%) and HIV unexposed 103/287
$(68.7 \%)$. Majority of the children were from mothers that were married 123/287 (82\%), unemployed 108/287 (72\%) with some primary level of education 88/287 (58.7\%) and from households comprising up to three children $88 / 287$ (58.7\%).

We calculated a cut-off of 4.7508 arbitrary rAU from pre-pandemic plasma of this cohort and detected 9/150 children aged 2 years old that were reactive for SARS-CoV-2 IgG above this cut-off giving a $6.0 \%$ seropositivity in this cohort comparable to the adult healthcare worker cohort. We found no association between background characteristics and SARS-CoV-2 IgG seropositivity (Table 2).

Table 1. Severe acute respiratory syndrome coronavirus 2 immunoglobulin G (SARS-CoV-2 IgG) seropositivity among healthcare workers by background characteristics.

\begin{tabular}{|c|c|c|c|}
\hline Characteristics & $\begin{array}{c}\text { \# of healthcare } \\
\text { workers (\% of total) }\end{array}$ & $\begin{array}{c}\#(\%) \\
\text { seropositive }\end{array}$ & $\begin{array}{c}\text { Fisher's exact } \\
\text { p-value }\end{array}$ \\
\hline Age (years): median (IQR) & $29(25,37)$ & & \\
\hline $18-25$ & $81(28.2)$ & $3(3.7)$ & \multirow{4}{*}{0.132} \\
\hline $26-35$ & $126(43.9)$ & $11(8.7)$ & \\
\hline $36-45$ & $45(15.7)$ & $7(15.6)$ & \\
\hline $45+$ & $35(12.2)$ & $2(5.7)$ & \\
\hline \multicolumn{4}{|l|}{ Sex } \\
\hline Female & $187(65.2)$ & $17(9.1)$ & \multirow{2}{*}{0.494} \\
\hline Male & $100(34.8)$ & $6(6.0)$ & \\
\hline \multicolumn{4}{|l|}{ Body mass index $\left(\mathrm{kg} / \mathrm{m}^{2}\right)$} \\
\hline Underweight & $17(5.9)$ & $0(0)$ & \multirow{4}{*}{0.192} \\
\hline Normal & $149(51.9)$ & $10(6.7)$ & \\
\hline Overweight & $114(39.7)$ & $13(11.4)$ & \\
\hline Missing & $7(2.4)$ & & \\
\hline \multicolumn{4}{|l|}{ Marital status } \\
\hline Single & $145(50.5)$ & $11(7.6)$ & \multirow{4}{*}{0.647} \\
\hline Married & $126(43.9)$ & $12(9.5)$ & \\
\hline Divorced/separated/ widowed & $13(4.5)$ & $0(0)$ & \\
\hline Missing & $3(1.1)$ & & \\
\hline \multicolumn{4}{|l|}{ HCW category } \\
\hline $\begin{array}{l}\text { Medical staff } \\
\text { Of these: } \\
\text { - Nurse/Mid-wife } \\
\text { - Lab Tech/Scientist } \\
\text { - Radiographer } \\
\text { - Other medical staff'1 }\end{array}$ & $\begin{array}{c}131(45.6) \\
90(68.7) \\
9(6.1) \\
3(2.3) \\
29(22.1)\end{array}$ & $\begin{array}{l}11(8.4) \\
8(8.9) \\
2(22.2) \\
1(33.3) \\
0(0)\end{array}$ & \multirow[t]{4}{*}{0.957} \\
\hline Support staff & $100(34.8)$ & $7(7.0)$ & \\
\hline Student & $53(18.5)$ & $4(7.6)$ & \\
\hline Missing & $3(1.1)$ & $1(33.3)$ & \\
\hline Total & $287(100)$ & $23(8.0)$ & \\
\hline
\end{tabular}

'Other medical staff include Clinical officers, Pharmacists, Anaesthetists, Dentists, Ophthalmologists, Public Health Officers and Environmental Health Scientists 


\begin{abstract}
Table 2. Severe acute respiratory syndrome coronavirus 2 immunoglobulin G (SARS-CoV-2 IgG) seropositivity in children aged 2 years old by background characteristics.
\end{abstract}

\begin{tabular}{|c|c|c|c|}
\hline Characteristics & $\begin{array}{c}\text { \# of children (\% } \\
\text { of total) }\end{array}$ & $\begin{array}{c}\text { \# (\%) } \\
\text { seropositive }\end{array}$ & $\begin{array}{c}\text { Fisher's exact } \\
\text { p-value }\end{array}$ \\
\hline Age (weeks): median (IQR) & $6(6,6)$ & & \\
\hline 5 & $26(17.3)$ & $2(7.7)$ & \multirow{2}{*}{0.861} \\
\hline 6 & $108(72.0)$ & $6(5.6)$ & \\
\hline $7+$ & $16(10.7)$ & $1(6.3)$ & \\
\hline \multicolumn{4}{|l|}{ Sex } \\
\hline Male & $84(56.0)$ & $7(8.3)$ & \multirow{2}{*}{0.299} \\
\hline Female & $66(44.0)$ & $2(3.0)$ & \\
\hline \multicolumn{4}{|l|}{ HIV exposure } \\
\hline Unexposed & $103(68.7)$ & $6(5.8)$ & \multirow{2}{*}{1.000} \\
\hline Exposed & $47(31.3)$ & $3(6.4)$ & \\
\hline \multicolumn{4}{|l|}{ Mother's marital status } \\
\hline Married & $123(82.0)$ & $5(4.1)$ & \multirow{2}{*}{0.057} \\
\hline Single & $27(18.0)$ & $4(14.8)$ & \\
\hline \multicolumn{4}{|l|}{ Mother's employment status } \\
\hline Unemployed & $108(72.0)$ & $7(6.5)$ & \multirow{2}{*}{1.000} \\
\hline Employed & $42(28.0)$ & $2(4.7)$ & \\
\hline \multicolumn{4}{|l|}{ Mother's educationallevel } \\
\hline None & $34(22.7)$ & $1(2.9)$ & \multirow{3}{*}{0.798} \\
\hline Primary & $88(58.7)$ & $6(6.8)$ & \\
\hline Secondary & $28(18.7)$ & $2(7.1)$ & \\
\hline \multicolumn{4}{|l|}{$\begin{array}{l}\text { Number of children in the } \\
\text { household }\end{array}$} \\
\hline $1-3$ & $88(58.7)$ & $3(3.4)$ & \multirow{3}{*}{0.194} \\
\hline $4-6$ & $55(36.7)$ & $6(10.9)$ & \\
\hline $7+$ & $7(4.7)$ & $0(0)$ & \\
\hline Total & $150(100)$ & $9(6.0)$ & \\
\hline
\end{tabular}

Significantly $(\mathrm{p}<0.001)$ higher mean arbitrary rAU was observed in post-pandemic plasma compared to pre-pandemic plasma in both healthcare workers and children (Figure 2).

Among the 23 SARS-CoV-2 IgG seropositives from the healthcare worker and 9 seropositives from the children cohort, we observed a significant difference in arbitrary rAU absorbance readings between pre- and post-pandemic plasma (Figure 3).

\section{Discussion}

We report comparable seroprevalence for SARS-CoV-2 S1 antigen in very young Zambian children and healthcare workers using a locally developed and optimized indirect ELISA assay. Direct comparison of pre- and post-pandemic plasma from the same individuals permits accurate evaluation of exposure to SARS-CoV-2 and avoids contaminating cross-reactive responses to other circulating human coronaviruses (hCoVs). Using an immunofluorescence assay, a recent analysis of pre-pandemic sera cohorts from sub-Saharan Africa estimated seroprevalence of up to $17.1 \%$ for SARS-CoV-2 nucleocapsid and up to $4 \%$ for spike trimer ${ }^{21}$. Up to $5 \%$ reactivity in pre-pandemic sera against spike S2 antigen was observed in SARS-CoV-2 unexposed UK individuals whereas no reactivity was detected against S1 antigen using a flow cytometry-based assay ${ }^{22}$. The 


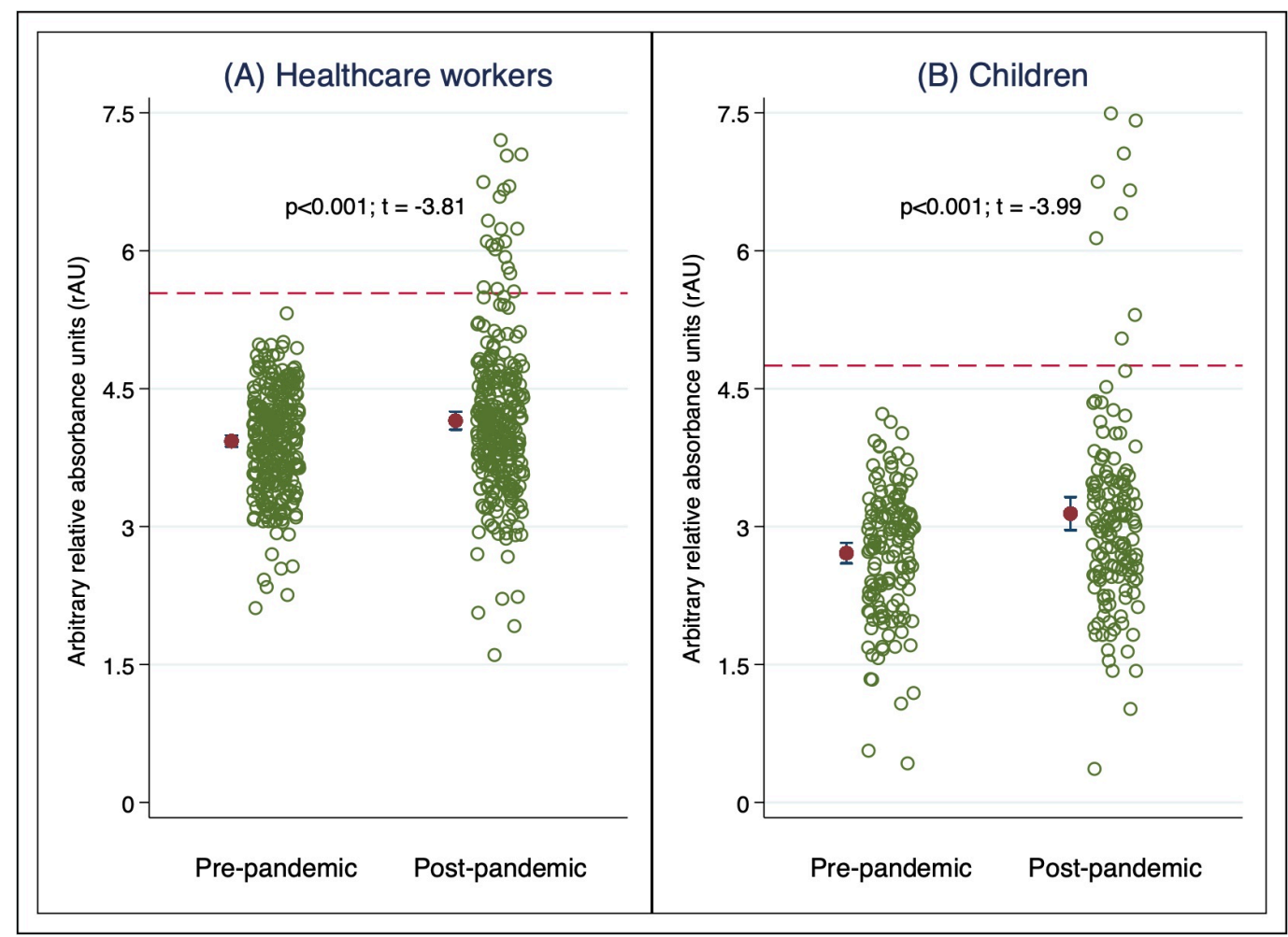

Figure 2. Distribution of natural-logarithm arbitrary relative absorbance units ( $r$ AU) pre-post COVID-19 pandemic period among healthcare workers (A) and children (B). The mean log-arbitrary rAU and 95\% confidence intervals are shown as range plots for the pre and post coronavirus disease 2019 (COVID-19) pandemic plasma for the healthcare workers $(n=287)$ and children $(n=150)$. The student t-test of equality of mean log-arbitrary rAU between the two periods for each cohort are shown. The red dashed line shows the calculated cut-off derived from the pre-pandemic samples of each cohort as the mean log-arbitrary rAU (+ 3 x standard deviations).

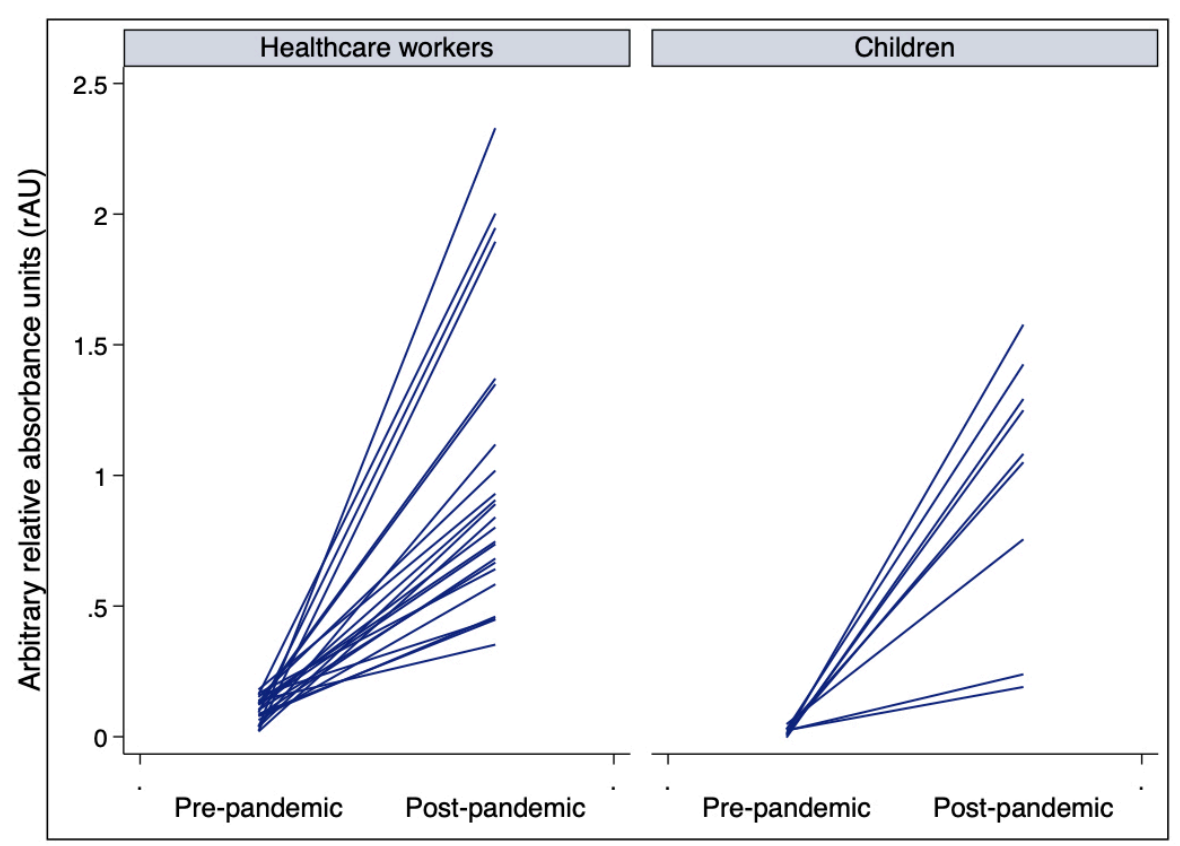

Figure 3. Distribution of natural log-arbitrary relative absorbance units ( $\mathrm{rAU}$ ) in the pre and post pandemic period among SARS-CoV-2 IgG seropositive children and healthcare workers. Pre-and post-pandemic plasma paired data points for arbitrary rAU are shown for severe acute respiratory syndrome coronavirus 2 (SARS-CoV-2) immunoglobulin $\mathrm{G}(\mathrm{IgG})$ seropositive healthcare workers $(\mathrm{n}=23$ ) and children $(n=9)$. 
selection of spike S1 protein for detection of SARS-CoV-2 in our assay therefore permits a further level of control for avoiding detection of potentially cross-reactive antibody responses $^{23,24}$. Further studies will assess the degree of exposure to circulating coronaviruses including hCoV-NL63, hCoV$229 \mathrm{E}, \mathrm{hCoV}-0 \mathrm{C} 43$ and hCoV-HKU1 to address the potential contribution of cross-protective immunity in the wider population. As circulating coronavirus infections are also acquired in early life $\mathrm{e}^{25,26}$, the acquisition of SARS-CoV-2 infection in very young children will be of particular interest, providing a potential opportunity to examine immunity to SARS-CoV-2 in children who are seronegative for circulating coronavirus strains.

The durability of anti-SARS-CoV-2 antibody responses also has important implications for estimates of seroprevalence and vaccine efficacy. Recent longitudinal studies have indicated significant and concerning decays in antibody responses to SARS-CoV-2 over time. Anti-nucleocapsid IgG titres were reported to decline by $50 \%$ within 3 months $^{27,28}$. However, IgG against spike trimer can be stable for at least 6 months ${ }^{27}$. The detection of antibody to spike $\mathrm{S} 1$ protein in our study therefore provides further confidence of the accuracy of our seroprevalence estimates, the longest likely intervals between infection and plasma sampling in our cohorts being between 8 months for healthcare workers and 12 months for young children. Longitudinal follow up of SARS-CoV-2 IgG seropositives presented in this study will inform the durability of antibody responses to spike $\mathrm{S} 1$ antigen in the two very different cohorts presented here. African countries including Zambia have begun to roll out vaccinations among their populations, with $>2,000$ individuals vaccinated in Zambia as of $19^{\text {th }}$ April $2021^{29}$. Serological assays such as the one presented here may prove useful in measuring longevity or waning of vaccine induced antibody responses and provide insights towards potential administration of booster doses.

At the beginning of August 2020, Zambia had recorded a cumulative 6,347 cases from a total of 85,560 tests conducted giving a $7.42 \%$ positivity rate, and cases continued rising to over 20,000 cases by December 2020 30,31 . During this period, we found $8 \%$ seroprevalence among healthcare workers and $6 \%$ seroprevalence among children within similar range to the country estimates. Our assay detects IgG responses which may be indicative of a previous and not an active SARS-CoV-2 infection and seropositive individuals in our study were at the time of sample collection non-hospitalised and otherwise asymptomatic, however, robust $\mathrm{IgG}$ responses can be detected as early as one week post exposure ${ }^{23}$. In any case, our results speak towards ongoing transmission within the healthcare setting and among very young children.

SARS-CoV-2 seropositivity among healthcare workers in Africa is variable across published reports which could be influenced by variable testing methods, healthcare worker cohorts studied and SARS-CoV-2 epidemiology. While we have reported $8 \%$ seroprevalence in healthcare workers using an in-house S1 ELISA, commercially available ELISA tests showed a $45 \%$ seroprevalence in Nigeria based on spike S-trimer protein ${ }^{32}$ while in Malawi this was $12.3 \%{ }^{33}$ based on $\mathrm{S} 2$ and $\mathrm{N}$ protein. The N, S2 and whole spike protein-based assays may overestimate exposure due to reported cross reactivity as opposed to the S1 antigen's superiority in distinguishing SARS-CoV-2 exposure. We found higher seropositivity among medical staff within the health facilities studied particularly among nurses and mid-wives. Reporting seroprevalence among healthcare workers distinguished by role within health facilities is important to better target health facility-based infection prevention and control measures.

Children typically experience less severe outcomes from SARS-CoV-2 infection, yet the reason for this is unknown. Many hypotheses have been suggested among which include exposure to hCoVs that imparts pre-existing cross-protective immunity, non-specific protective effects of live vaccines, differential distribution of and higher protective serum circulating angiotensin-converting enzyme 2 (ACE2), the SARS-CoV-2 receptor $^{34,35}$. While the present study is limited in that we did not test for exposure and potential cross reactivity to seasonal hCoVs, our data has not shown any antibodies reactive to our ELISA target from pre-pandemic samples. Nonetheless, we have shown that post-pandemic, children are just as likely to get infected as adults and infections in children as young as 2 years old are prevalent and have the potential to be transmitted widely within communities. These infections, being asymptomatic have far reaching implications for transmissions within households and schools, and therefore the paediatric population needs to be considered in large scale screening and immunosurveillance campaigns. In the very young children like those included in our study, acquisition of infection from mothers is an important route of infection calling for the need to conduct research on transmission dynamics and control measures within mother-child cohorts. Additional research is also required to address unanswered questions on reasons for the lower disease severity in children.

Our study was limited in that our assay measured IgG which may not reflect active infection, utilized samples from another study which was not powered for this study's aim and lacked comparison to available commercial tests. However, we are confident in our results as the pair of pre and post pandemic samples accounts for possible cross reactivity as a confounder, with the S1 antigen having little to no reported serological cross reactivity with other hCoVs. We tested a specific population of healthcare workers from a single district. The seroprevalence results may not be generalizable to the overall healthcare worker population across different types of health facilities and districts having different levels of catchment population, infection control capacity and infrastructure. A larger and more representative sample and powered study may be required to accurately assess this. However, our data provides an estimate of seroprevalence in this specific group. We tested seroprevalence in children who were recruited under an ongoing trial and remained in follow-up. Children were enrolled as they randomly attended the health facility and therefore results could be generalizable in this specific age 
group. However, differences in characteristics of children that remained in follow up as opposed to those who did not and were therefore not tested could potentially influence estimates and may limit this generalization.

\section{Conclusions}

Establishment of the SARS-CoV-2 antibody assay in-country has potential to create avenues for epidemiological immunosurveillance studies to determine SARS-CoV-2 exposure in the local communities and specific populations. In our study, we have shown that children's exposure to SARS-CoV-2 infection is comparable to high-risk adults highlighting the importance of this paediatric population when considering infection control measures. Such significant exposure among very young children should inform targeting households in addition to infection prevention and control measures being applied at health facility level.

\section{Data availability}

Figshare: Comparable exposure to SARS-CoV-2 in young children and healthcare workers in Zambia. https://doi.org/ 10.6084/m9.figshare.14237285.v220.
This project contains the following underlying data:

- $\quad$ SARS_Data_Dictionery.pdf

- $\quad$ SARS Child dataset.csv

- $\quad$ SARS HCW dataset.csv

Data are available under the terms of the Creative Commons Attribution 4.0 International License (CC BY 4.0)

\section{Acknowledgements}

We would like to acknowledge the children and healthcare workers that comprised the study cohorts and the Research Physicians, Nurses, Field Assistants, Data Associates and Laboratory support staff under the CIDRZ Enteric and Vaccines Research Unit that were involved under the Hepatitis B and rotavirus vaccine studies. We also acknowledge the University of Oxford and European Developing Countries Clinical Trials Partnership for respectively supporting the work that recruited the cohorts of health care workers and young children in the existing studies.
1. WHO Coronavirus Disease (COVID-19) Dashboard. World Health Organization. 2021; [cited 12th April 2021]. Reference Source

2. Africa CDC Dashboard: Coronavirus Disease 2019 (COVID-19). Africa CDC 2021; [cited 12th April 2021]. Reference Source

3. ZNPHI: Zambia COVID-19 Situational Report No. 1. 2020 Reference Source

4. Wu F, Zhao S, Yu B, et al.: A new coronavirus associated with human respiratory disease in China. Nature. 2020; 579(7798): 265-9. PubMed Abstract | Publisher Full Text | Free Full Text

5. Corman VM, Landt O, Kaiser M, et al.: Detection of 2019 novel coronavirus (2019-nCoV) by real-time RT-PCR. Euro Surveill. 2020; 25(3): 2000045. PubMed Abstract | Publisher Full Text | Free Full Text

6. Funk $C D$, Laferrière $C$, Ardakani $A$ : A Snapshot of the Global Race for Vaccines Targeting SARS-CoV-2 and the COVID-19 Pandemic. Front Pharmacol. 2020; 11(937): 937.

PubMed Abstract | Publisher Full Text | Free Full Text

7. Jayamohan $\mathrm{H}$, Lambert CJ, Sant HJ, et al.: SARS-CoV-2 pandemic: a review of molecular diagnostic tools including sample collection and commercial response with associated advantages and limitations. Anal Bioanal Chem. 2020; 413(1): 49-71.

PubMed Abstract | Publisher Full Text | Free Full Text

8. Yong SEF, Anderson DE, Wei WE, et al.: Connecting clusters of COVID-19: an epidemiological and serological investigation. Lancet Infect Dis. 2020; 20(7): 809-15.

PubMed Abstract | Publisher Full Text | Free Full Text

9. Alandijany TA, El-Kafrawy SA, Tolah AM, et al:: Development and Optimization of In-house ELISA for Detection of Human IgG Antibody to SARS-CoV-2 Full Length Spike Protein. Pathogens. 2020; 9(10): 803. PubMed Abstract | Publisher Full Text | Free Full Text

10. Peterhoff $D$, Glück V, Vogel M, et al.: A highly specific and sensitive serological assay detects SARS-CoV-2 antibody levels in COVID-19 patients that correlate with neutralization. Infection. 2021; 49(1): 75-82. PubMed Abstract | Publisher Full Text | Free Full Text

11. ZNPHI: Zambia COVID-19 Situational Report No. 75. 2020. Reference Source

12. Wu Z, McGoogan JM: Characteristics of and Important Lessons From the Coronavirus Disease 2019 (COVID-19) Outbreak in China: Summary of a Report of 72314 Cases From the Chinese Center for Disease Control and Prevention. JAMA. 2020; 323(13): 1239-42.

PubMed Abstract | Publisher Full Text
13. Poline J, Gaschignard J, Leblanc C, et al.: Systematic SARS-CoV-2 screening at hospital admission in children:a French prospective multicenter study. Clin Infect Dis. 2020; ciaa1044.

PubMed Abstract | Publisher Full Text | Free Full Text

14. Han MS, Choi EH, Chang SH, et al.: Clinical Characteristics and Viral RNA Detection in Children With Coronavirus Disease 2019 in the Republic of Korea. JAMA Pediatr. 2021; 175(1): 73-80.

PubMed Abstract | Publisher Full Text | Free Full Text

15. Demonstration Project on Health Care Worker Protection Against Hepatitis B in Kalulushi District. NCT04072211 ClinicalTrials.gov: U.S. National Library of Medicine. Reference Source

16. 2010 CENSUS OF POPULATION AND HOUSING: Population and Demographic Projections 2011-2035. 2013. Reference Source

17. Fleiss JL, Levin B, Paik MC: Statistical Methods for Rates and Proportions. 3rd Edition. 2003. Reference Source

18. Newcombe RG: Two-sided confidence intervals for the single proportion: comparison of seven methods. Stat Med. 1998; 17(8): 857-72. PubMed Abstract | Publisher Full Text

19. A randomized controlled trial of two versus three doses of Rotarix vaccine for boosting and longevity of vaccine immune responses in Zambia. PACTR201804003096919: Pan African Clinical Trials Registry. Reference Source

20. Laban N: Comparable exposure to SARS-CoV-2 in young children and healthcare workers in Zambia. 2021

https://figshare.com/articles/dataset/SARS Data_Dictionary_pdf/14237285

21. Tso FY, Lidenge SJ, Peña PB, et al.: High prevalence of pre-existing serological cross-reactivity against severe acute respiratory syndrome coronavirus-2 (SARS-CoV-2) in sub-Saharan Africa. Int J Infect Dis. 2021; 102: 577-83. PubMed Abstract | Publisher Full Text | Free Full Text

22. Ng KW, Faulkner N, Cornish GH, et al:: Preexisting and de novo humoral immunity to SARS-CoV-2 in humans. Science. 2020; 370(6522): 1339-1343. PubMed Abstract | Publisher Full Text | Free Full Text

23. Algaissi A, Alfaleh MA, Hala S, et al:: SARS-CoV-2 $\mathbf{S 1}$ and $\mathbf{N}$-based serological assays reveal rapid seroconversion and induction of specific antibody response in COVID-19 patients. Sci Rep. 2020; 10(1): 16561. PubMed Abstract | Publisher Full Text | Free Full Text

24. Tian Y, Lian C, Chen Y, et al.: Sensitivity and specificity of SARS-CoV-2 S1 subunit in COVID-19 serology assays. Cell Discov. 2020; 6(1): 75 PubMed Abstract | Publisher Full Text | Free Full Text 
25. Zhou W, Wang W, Wang H, et al:: First infection by all four non-severe acute respiratory syndrome human coronaviruses takes place during childhood. BMC Infect Dis. 2013; 13(1): 433. PubMed Abstract | Publisher Full Text | Free Full Text

26. Dijkman R, Jebbink MF, El Idrissi NB, et al.: Human coronavirus NL63 and 229E seroconversion in children. J Clin Microbiol. 2008; 46(7): 2368-73. PubMed Abstract | Publisher Full Text | Free Full Text

27. Lumley SF, Wei J, O'Donnell D, et al.: The duration, dynamics and determinants of SARS-CoV-2 antibody responses in individual healthcare workers. medRxiv. 2020; 2020.11.02.20224824. Publisher Full Text

28. Bolotin S, Tran V, Osman S, et al.: SARS-CoV-2 seroprevalence survey estimates are affected by anti-nucleocapsid antibody decline. medRxiv. 2020; 2020.09.28.20200915. Publisher Full Text

29. Zambia COVID-19 Statistics - Daily Status Updates. Ministry of Health; 2021.

30. ZNPHI: Zambia COVID Situational Report No. 75. 2nd August 2020. 2020 Reference Source

31. COVID-19 Dashboard by the Center for Systems Science and Engineering
(CSSE) at Johns Hopkins University (JHU). Johns Hopkins University \& Medicine. 2021; [cited 14th January 2020].

Reference Source

32. Olayanju O, Bamidele O, Edem F, et al.: SARS-CoV-2 Seropositivity in Asymptomatic Frontline Health Workers in Ibadan, Nigeria. Am J Trop Med Hyg. 2021; 104(1): 91-4.

PubMed Abstract | Publisher Full Text | Free Full Text

33. Chibwana MG, Jere KC, Kamng'ona R, et al.: High SARS-CoV-2 seroprevalence in Health Care Workers but relatively low numbers of deaths in urban Malawi. medRxiv. 2020; 2020.07.30.20164970. PubMed Abstract | Publisher Full Text | Free Full Text

34. Zimmermann $\mathrm{P}$, Curtis N: Why is CovID-19 less severe in children? A review of the proposed mechanisms underlying the age-related difference in severity of SARS-CoV-2 infections. Arch Dis Child. 2020; archdischild-2020-320338.

PubMed Abstract | Publisher Full Text

35. Ciaglia E, Vecchione C, Puca AA: COVID-19 Infection and Circulating ACE2 Levels: Protective Role in Women and Children. Front Pediatr. 2020; 8: 206. PubMed Abstract | Publisher Full Text | Free Full Text 


\section{Open Peer Review}

\section{Current Peer Review Status: ? ?}

\section{Version 1}

Reviewer Report 27 September 2021

https://doi.org/10.21956/wellcomeopenres.18481.r45560

(C) 2021 Muhsen K. This is an open access peer review report distributed under the terms of the Creative Commons Attribution License, which permits unrestricted use, distribution, and reproduction in any medium, provided the original work is properly cited.

\section{? Khitam Muhsen}

Department of Epidemiology and Preventive Medicine, School of Public Health, Sackler Faculty of Medicine, Tel Aviv University, Tel Aviv, Israel

This is an important manuscript that presents results from Zambia related to the development of SARS-CoV-2 ELISA for the detection of anti-S IgG antibodies. The authors applied the newly developed assay to sera of health care workers and children and assessed sero-positivity to SARSCoV-2. Evidence on SARS-CoV-2 in children is relatively limited especially from low income countries.

I have a few minor comments.

Methods section page 3:

"The sample size was adjusted by $25 \%$ to control for losses to follow-up and secondary analyses". Please change "adjusted" to "increased".

Laboratory methods:

Page 4: please add the values of the sensitivity and specificity of the commercial SARS-CoV-2 Spike/S1-His recombinant protein (Cat 40591-V08H, Sino Biologicals, China). Please add details on the purpose of using this kit.

In-house ELISA:

How much time it takes to run the assay until a result is available? Please add this summary information.

Reliability analysis:

Were the same samples tested more than once? If so please report these results.

Regarding cutoff value to determine positive results in in-house ELISA, is there any range of equivocal results? 
Results section:

Studies from some high income countries such as Israel, Greece and Denmark showed low seroprevalence of SARS-CoV-2 antibodies during the early waves of the COVID-19 pandemic in these countries (1-8\%), and it was related to exposure to COVID-19 patients. See the following references including our work from Israel (Muhsen et al. Front Med 2021).

Can the author comment on their findings of $8 \%$ in health care workers in Zambia compared to high income countries and low-middle income countries, especially in relation to exposure to Covid-19 patients.

The $6 \%$ positivity in children ages 2 years seems high.

The different cutoff values used for different populations limits the comparability between children and health care workers in this study. Figure 2 shows a few outlier observations in children that may have led to lower cutoff value, and 2 observations were around the cutoff value might be in fact negative if the same cutoff of adults was used. It is recommended to define a range of equivocal results to avoid overestimation.

\section{References:}

Muhsen K, Schwaber MJ, Bishara J, et al. Sero-Prevalence and Sero-Incidence of Antibodies to SARS-CoV-2 in Health Care Workers in Israel, Prior to Mass COVID-19 Vaccination. Front Med (Lausanne). 2021 Jun 24;8:689994. doi: 10.3389/fmed.2021.

Psichogiou M, Karabinis A, Pavlopoulou ID, et al. Antibodies against SARS-CoV-2 among health care workers in a country with low burden of COVID-19. PLoS ONE. (2020) 15:e0243025. 10.1371/journal.pone.0243025

Iversen K, Bundgaard H, Hasselbalch RB, et al. Risk of COVID-19 in health-care workers in Denmark: an observational cohort study. Lancet Infect Dis. (2020) 20:1401-8. 10.1016/S14733099(20)30589-2

\section{References}

1. Muhsen K, Schwaber MJ, Bishara J, Kassem E, et al.: Sero-Prevalence and Sero-Incidence of Antibodies to SARS-CoV-2 in Health Care Workers in Israel, Prior to Mass COVID-19 Vaccination. Front Med (Lausanne). 2021; 8: 689994 PubMed Abstract | Publisher Full Text

2. Psichogiou M, Karabinis A, Pavlopoulou ID, Basoulis D, et al.: Antibodies against SARS-CoV-2 among health care workers in a country with low burden of COVID-19.PLoS One. 2020; 15 (12): e0243025 PubMed Abstract | Publisher Full Text

3. Iversen K, Bundgaard H, Hasselbalch R, Kristensen J, et al.: Risk of COVID-19 in health-care workers in Denmark: an observational cohort study. The Lancet Infectious Diseases. 2020; 20 (12): 1401-1408 Publisher Full Text

Is the work clearly and accurately presented and does it cite the current literature? Yes

Is the study design appropriate and is the work technically sound? 
Yes

Are sufficient details of methods and analysis provided to allow replication by others? Yes

If applicable, is the statistical analysis and its interpretation appropriate? Partly

Are all the source data underlying the results available to ensure full reproducibility? Yes

Are the conclusions drawn adequately supported by the results? Partly

Competing Interests: No competing interests were disclosed.

Reviewer Expertise: Epidemiology of infectious diseases, including COVID-19, Sero-epidemiology

I confirm that I have read this submission and believe that I have an appropriate level of expertise to confirm that it is of an acceptable scientific standard, however I have significant reservations, as outlined above.

Reviewer Report 09 July 2021

https://doi.org/10.21956/wellcomeopenres.18481.r44538

(C) 2021 Mayne E. This is an open access peer review report distributed under the terms of the Creative Commons Attribution License, which permits unrestricted use, distribution, and reproduction in any medium, provided the original work is properly cited.

\section{Elizabeth Mayne}

Department of Immunology, Faculty of Health Sciences, University of Witwatersrand, Johannesburg, South Africa

This is an interesting paper which appears to have 3 objectives:

1. The development of an in-house elisa assay against the spike protein of SARS-CoV2

2. The seroprevalence in healthcare workers

3. The seroprevalence in children

Each of these objectives could have stood independently and I feel the conflation of the three results in some confusion.

With respect to the assay development, it is well-described. It would be improved, however, by a more rigorous evaluation (for example, comparison with a gold standard like PCR and with commercially available assays). 
The cohorts used were both convenient, but there is not description of possible symptomatology.

It is unclear from both the introduction and the conclusion as to why socioeconomic status, marital status or BMI was included in the description. There are some significant omissions, including the absence of description of any symptoms. The healthcare worker cohort should be stratified according to degree of patient contact. The age of the children is unclear with respect to the blood draws and this should be clarified

Figure 2 and 3 do not enhance the test and should be removed.

This work utilises two existing cohorts - 1 paediatric (a rotavirus vaccine boost trial) and 1 adult (healthcare workers or students working in healthcare). These are interesting and potentially useful cohorts but I think they should be separated. With respect to the paediatric cohort, I have the following questions:

1. Was the age reported the age at the first blood draw? If yes, how old were the children who were included with a second blood draw?

2. Please justify the inclusion of maternal socioeconomic status. Is there any indication that the Zambian experience of SARS-CoV2 is affected by either educational or marital status

3. Were there any symptoms reported by the mothers?

For the healthcare worker:

1. Exposure differs amongst different categories of healthcare - please clarify what the "other healthcare workers" were, what the students were

The assay development is interesting. It would benefit, however, from comparison (if only in a subpopulation) with a commercially validated assay

A logistic regression may be of use when comparing the different multiple factors rather than a Fishers exact test. The calculation of the relative absorption units is difficult to follow and may benefit from more thorough statistical review. Figure 2 and 3 are not helpful to the text. It is to be expected that more participants will test positive following the pandemic spread so comparing the pre- and post-pandemic relative absorption units and saying that they are higher after the participants were exposed is not meaningful in this setting.

Is the work clearly and accurately presented and does it cite the current literature? Yes

Is the study design appropriate and is the work technically sound? Partly

Are sufficient details of methods and analysis provided to allow replication by others? Yes

If applicable, is the statistical analysis and its interpretation appropriate? 
Partly

Are all the source data underlying the results available to ensure full reproducibility? Yes

Are the conclusions drawn adequately supported by the results?

Partly

Competing Interests: No competing interests were disclosed.

Reviewer Expertise: Immunology, haematology

I confirm that I have read this submission and believe that I have an appropriate level of expertise to confirm that it is of an acceptable scientific standard, however I have significant reservations, as outlined above. 